\title{
On the equivalence of pulsed and continuous ultrasound in producing temperature elevations resulting from absorption
}

\author{
James L. Drewniak† and Floyd Dunn \\ Bioacoustics Research Laboratory, University of Illinois, \\ Urbana, Illinois 61801, USA
}

(Received 30 August 1991)

\begin{abstract}
It is commonly accepted that the temperature elevation in biological specimens resulting from ultrasonic absorption will be the same for both continuous and pulsed ultrasound fields for identical spatial-peak, temporal-average intensities. The rate of heat generation per unit volume is typically determined by time averaging the square of the pressure waveform divided by $\rho c_{o}$ over the pulse repetition frequency. The assumption that pulsed and continuous ultrasound fields produce the same temperature elevation for identical spatial-peak, temporal-average intensities is examined from an alternate perspective and is found to be consistent with the above usual practice.
\end{abstract}

Keywords: Ultrasound, Absorption, Temperature elevation, Pulsed and CW equivalence

PACS number: 43. 20. Hq, 43. 35. Wa

\section{INTRODUCTION}

It is well-documented that one mechanism of physical damage to living systems exposed to ultrasound is temperature elevation resulting from absorption of the wave in the body of the tissue. ${ }^{1-3)}$ The temperature increase resulting from exposure to ultrasound is thus of concern in medical applications of ultrasound. Both continuous wave $(\mathrm{cw})$ and pulsed ultrasound fields have been employed in ultrasound bioeffects studies where heat generation is of concern, and in studies of heat generation in tissue and tissue-mimmicking media. Often the results are reported in terms of the spatial-peak, temporal-average (SPTA) intensity $I_{\mathrm{SPTA}}$. The choice of whether $c w$ or pulsed ultrasound is employed in a particular application may be determined by the available resources. Physical limitations may also determine the choice, as for example, in studies of the temperature elevation resulting from ultrasonic

\footnotetext{
$\dagger$ Present address: Department of Electrical Engineering University of Missouri-Rolla, MO 65401
}

absorption in a nonlinear field, where SPTA intensities required to generate the desired nonlinear field produce excessive temperature increases. One method for reducing the temperature increase produced by a nonlinear field is the use of pulsed ultrasound. It seems intuitive, and indeed is the accepted practice, that the temperature elevation resulting from $\mathrm{cw}$ and pulsed ultrasound will be identical for identical $I_{\text {SPTA }}$, ultrasound field profiles, and harmonic generation. Indeed, reported measurements of heat generation in agar gels resulting from absorption in a nonlinear ultrasound field, which assumes the equivalence of pulsed and $c w$ ultrasound with respect to heat generation, suggests this to be the case. ${ }^{4}$

The assumption of the equivalence of pulsed and $c w$ ultrasound in producing temperature elevations in an absorbing medium is examined in this paper from another perspective and shows it to be in agreement with the accepted belief that the temperature elevation resulting from absorption of identical pulsed and $c w$ ultrasound fields of like 
$I_{\mathrm{SPTA}}$ will be the same.

\section{RESULTS}

One representation for the solution to the heat equation, when considering the temperature elevation resulting from absorption in tissue exposed to ultrasound, is given by the convolution of the equivalent heat source and the appropriate Green's function over the absorbing volume $V$ as ${ }^{5,6}$ )

$$
\begin{aligned}
T(\overline{\boldsymbol{r}}, t)= & \frac{q_{v o}}{\rho C_{p}} \int_{0}^{t} d \zeta F(\zeta) \int_{V} d \overline{\boldsymbol{r}}^{\prime} f\left(\overline{\boldsymbol{r}}^{\prime}\right) \\
& \cdot G\left(\overline{\boldsymbol{r}}-\overline{\boldsymbol{r}}^{\prime}, t-\zeta\right)
\end{aligned}
$$

where $\rho C_{p}$ is the volumetric heat capacity, $q_{v o}$ is the rate of heat generation per unit volume, $t$ is the time, $F(t)$ is the temporal variation of the heat source, $f(\overline{\boldsymbol{r}})$, is the distributed heat source, $G\left(\overline{\boldsymbol{r}}-\overline{\boldsymbol{r}}^{\prime}\right)$ is the appropriate Green's function, and $\overline{\boldsymbol{r}}$ and $\overline{\boldsymbol{r}}^{\prime}$ are the observation and source coordinates, respectively. In the case of soft tissue, the thermal properties are often taken to be those of water and the Green's function for an infinite, homogeneous medium is

$$
G\left(\overline{\boldsymbol{r}}-\overline{\boldsymbol{r}}^{\prime}, t-\zeta\right)=\frac{\exp \left[-\left|\overline{\boldsymbol{r}}-\overline{\boldsymbol{r}}^{\prime}\right|^{2} / 4 \kappa(t-\zeta)\right]}{[4 \pi \kappa(t-\zeta)]^{3 / 2}}
$$

where $\kappa=1.5 \times 10^{-3} \mathrm{~cm}^{3} / \mathrm{s}$ is the thermal diffusivity of water. The rate of heat generation for energy deposition resulting from ultrasonic absorption is the time average $\left\langle q_{v}\right\rangle$ over the duration of the exposure. An example is shown in Fig. 1 of the temperature increase obtained from Eq. (1) at the center of a homogeneous tissue specimen of thickness $2 \mathrm{~mm}$ and $1 \mathrm{~cm} \times 1 \mathrm{~cm}$ transverse tissue dimensions, exposed to an incident Gaussian-shaped ultrasound beam with a $1 \mathrm{~mm}$ half-power beamwidth and $\left\langle q_{v}\right\rangle$

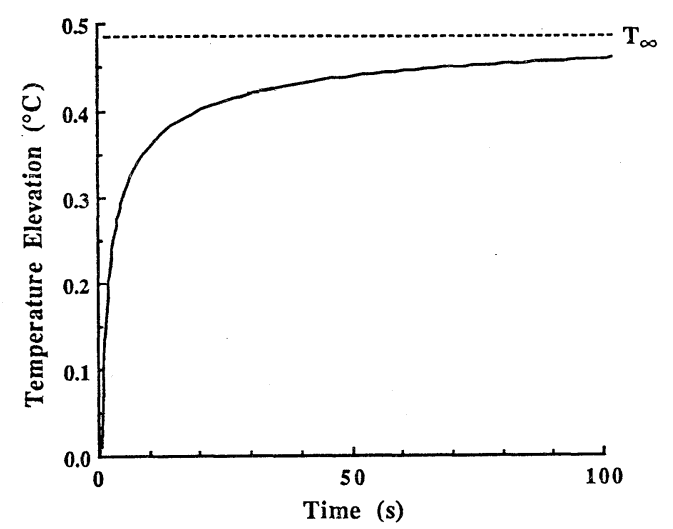

Fig. 1 Temperature elevation in a homogeneous medium calculated from Eq. (1).
$=1 \mathrm{~W} / \mathrm{cm}^{3}$. Reported comparisons between measured temperature elevations in soft tissue and calculations employing Eq. (1) exhibit good agreement. ${ }^{4,7-9)}$

The time average rate of heat generation in a relaxing medium with zero shear viscosity for a $\mathrm{cw}$ exposure has been shown to be given by ${ }^{10,11)}$

$$
\left\langle q_{v}\right\rangle_{\mathrm{cw}}=\alpha \frac{p_{o}^{2}}{\rho c_{o}}
$$

where $\alpha$ is the infinitestimal amplitude ultrasonic absorption coefficient, $p_{o}$ is the pressure amplitude of the ultrasonic field, $\rho$ is the density, and $c_{o}$ is the infinitesimal amplitude speed of sound. Heat generation in a nonlinear field will be discussed later. Equation (2) relating the time-average volumetric rate of heat generation to the peak acoustic pressure $p_{o}$ in a $c w$ field is, in fact, more general and can be written as ${ }^{12)}$

$$
\left\langle q_{v}\right\rangle=2 \alpha \frac{\left\langle p^{2}(t)\right\rangle}{\rho c_{o}}
$$

where $p(t)$ is the acoustic pressure at a specified location. For a traveling plane wave, the square of the pressure amplitude at a particular location in the ultrasound field, divided by $\rho c_{o}$ and integrated over the period is the temporal average intensity, and for a $c w$ exposure, $(1 / 2)\left(p_{o}{ }^{2} / \rho c_{o}\right)=I_{\mathrm{TA}}$. The timeaverage rate of heat generation per unit volume is then the familiar expression $\left\langle q_{v}\right\rangle_{\mathrm{CW}}=2 \alpha I_{\mathrm{TA}}$.

Since the thermal response time constant is typically much generater than the pulse width and the period between pulses for typical pulsed fields, it is common to assume a unit step temporal variation $F(t)=U(t)$ in Eq. (1) and determine $\left\langle q_{v}\right\rangle$ as

$$
\left\langle q_{v}\right\rangle=2 \alpha \frac{\left\langle p^{2}\right\rangle}{\rho c_{o}}=2 \alpha \frac{1}{t_{\mathrm{PRF}}} \int_{0}^{t_{\mathrm{PRF}}} \frac{p^{2}(t)}{\rho c_{o}} d t
$$

where $t_{P R F}$ is the reciprocal of the pulse repetition frequency $(P R F)$. It should be noted that the time average is taken over the pulse width, $t_{\mathrm{PW}}$, plus the off time until the next pulse. The integral term in Eq. (4) is defined for medical ultrasound fields as $t_{\mathrm{PW}} I_{\mathrm{PA}}$, where $I_{\mathrm{PA}}$ is the pulse-average intensity. The heat generation rate per unit volume for the pulsed field is then

$$
\left\langle q_{v}\right\rangle=2 \alpha P R F \times t_{\mathrm{PW}} \times I_{\mathrm{PA}}=2 \alpha I_{\mathrm{TA}}
$$

An alternate perspective is to consider the pulse train of the ultrasound as the temporal variation in the rate of energy deposition $F(t)$, and $\left\langle q_{v}\right\rangle$ as the time-average rate of heat generation per unit volume 


\section{J. L. DREWNIAK and F. DUNN: PULSED AND CW ULTRASOUND TEMPERATURE ELEVATION}

over a single pulse $\left\langle q_{v}\right\rangle_{\mathrm{P}}$. It is shown below that these two perspectives are consistent.

It is assumed for the sake of mathematical simplicity that a tissue specimen is irradiated by a singlebeam, traveling wave, $c w$ ultrasound field, and that the temperature elevation observed at the location of maximum heat generation can be adequately approximated by a finite sum of simple exponentials of the form $(1-\exp (-t / \tau))$. The temperature increase as a function of time in the specimen for a $c w$ or unit step exposure, i.e., $F(t)=U(t)$, is then taken to be

$$
\begin{aligned}
T_{c w}(t) & =T(\overline{\boldsymbol{r}}, t) \\
& =\frac{\left\langle q_{v}\right\rangle_{c \mathrm{w}}}{\rho C_{p}} t \int_{0}^{1} d \xi \int_{V} d \overline{\boldsymbol{r}}^{\prime} f\left(\overline{\boldsymbol{r}}^{\prime}\right) G\left(\overline{\boldsymbol{r}}-\overline{\boldsymbol{r}}^{\prime}, t \xi\right) \\
& \approx T_{\infty} \sum_{n=1}^{N} a_{n}\left(1-\exp \left(-t / \tau_{n}\right)\right)
\end{aligned}
$$

where a change of variables $t-\zeta=t \xi$ has been made to place the dimensions outside of the integrals. The value $T_{\infty}$ is the temperature increase in the tissue at steady state, i.e., as $t \rightarrow \infty$. From Eq. (5), $T_{\infty}$ is given by

$$
T_{\infty}=\frac{\left\langle q_{v}\right\rangle_{\mathrm{cw}}}{\rho C_{p}} \phi
$$

where $\phi$ is the limiting value of the product of $t$ and the time integral in Eq. (5) as $t \rightarrow \infty$ and has units of seconds. Upon using Eq. (2) together with Eq. (5), and making the substitution $(1 / 2)\left(p_{o}{ }^{2} / \rho c_{o}\right)=I_{\mathrm{SPTA}}$, where $I_{\mathrm{SPTA}}$ denotes the spatial-peak, temporalaverage intensity, $T_{\infty}$ is

$$
T_{\infty}=\frac{\left\langle q_{v}\right\rangle_{\mathrm{cW}}}{\rho C_{p}} \phi=\frac{\alpha}{\rho C_{p}} \frac{p_{o}{ }^{2}}{\rho c_{o}} \phi=\frac{2 \alpha}{\rho C_{p}} I_{\mathrm{SPTA}} \phi
$$

The temperature increase for a $c w$ exposure is then approximated by

$$
T_{c w}(t)=\frac{2 \alpha}{\rho C_{p}} I_{\mathrm{SP} \mathrm{IA}} \phi \sum_{n=1}^{N} a_{n}\left(1-\exp \left(-t / \tau_{n}\right)\right)
$$

It has been shown analytically from Eq. (1) that the temperature elevation in soft tissue exposed to an ultrasound beam at the location of maximum heat generation, typically the beam intensity maximum, is linear for short times $t .^{13)}$ This is also observed experimentally for soft and hard tissues, ${ }^{14,15)}$ and is the basis for measuring the ultrasonic absorption coefficient in soft tissues and liquids. ${ }^{14)}$ Thus, the coefficients $a_{n}$ in the summation in Eq. (5) and Eq. (7) must be constrained to give $T_{c w}$ the correct behavior as $t \rightarrow 0$, as well as for $t \rightarrow \infty$.

The heat equation is a linear time invariant dif- ferential equation, hence, standard mathematical developments can be employed to determine the temperature increase resulting from a pulsed ultrasound field exposure once the $c w$ field exposure is known for spatially identical ultrasound fields, i.e., identical beam shapes (and harmonic development). The temperature increase for the pulsed field is simply the convolution of the pulse train with the thermal impulse response. The temperature increase resulting from a $c w$ exposure with a heat generation rate of $\left\langle q_{v}\right\rangle_{\mathrm{cw}}=1 \mathrm{~W} / \mathrm{cm}^{2}$ (hence $T_{\infty}=$ $\phi\left(\rho c_{p}\right)$ is the thermal unit step response. The thermal impulse response $T_{\hat{\delta}}(t)$, which is the derivative of the $c w$ response given in Eq. (5) is then

$$
\frac{d T_{c w}(t)}{d t}=T_{\tilde{o}}(t)=T_{\infty} \frac{a_{n}}{\tau_{n}} \exp \left(-t / \tau_{n}\right)
$$

Only a single term from the sum in Eq. (5) has been shown in Eq. (8) for simplicity and clarity in the following development.

Typical medical ultrasound fields employ pulse widths of the order of microseconds and pulse repetition frequencies in the kilohertz range. Such a pulse train can be described by

$$
F(t)=\left\langle q_{v}\right\rangle_{\mathrm{p}} \sum_{m=0}^{M-1} u\left(t-m t_{\mathrm{PRF}}\right)-u\left(t-m t_{\mathrm{PRF}}-t_{\mathrm{PW}}\right)
$$

where $t_{\mathrm{PW}}$ is the pulse width in seconds, $t_{\mathrm{PRF}}$ is the reciprocal of the PRF, $\left\langle q_{v}\right\rangle_{p}$ is the time average volumetric rate of heat generation over a single pulse, and $M$ is the number of pulses in the exposure. Proceeding with the standard linear systems theory development, the temperature elevation resulting from the pulsed ultrasound field is

$$
\begin{aligned}
T_{\mathrm{p}}(t) & =F(t) *\left(\frac{d T_{c w}}{d t}\right)=F(t) * T_{\delta}(t) \\
& =\int_{0}^{t} F(\xi) T_{\delta}(t-\xi) d \xi
\end{aligned}
$$

where the asterisk denotes the convolution operation. The temperature elevation resulting from ultrasonic absorption in a pulsed field $T_{p}(t)$ is then given by

$$
\begin{aligned}
T_{p}(t)= & \left\langle q_{v}\right\rangle_{\mathrm{p}} \int_{0}^{t} d \xi \sum_{m=0}^{M-1}\left[u\left(\xi-m t_{\mathrm{PRF}}\right)\right. \\
& \left.-u\left(t-m t_{\mathrm{PRF}}-t_{\mathrm{pw}}\right)\right] T_{\delta}(t-\xi)
\end{aligned}
$$

Equation (11) is general and can be used with any analytically or experimentally determined thermal impulse response. Substituting the impulse re- 
sponse given by Eq. (8) into Eq. (11), and interchanging the order of integration and summation yields

$$
\begin{aligned}
T_{p}(t)= & \left\langle q_{v}\right\rangle_{\mathrm{p}} T_{\infty} \frac{a_{n}}{\tau_{n}} \sum_{m=0}^{M-1} \int_{0}^{t}\left[u\left(\xi-m t_{\mathrm{PRF}}\right)\right. \\
& \left.-u\left(t-m t_{\mathrm{PRF}}-t_{\mathrm{PW}}\right)\right] \exp \left(-(t-\xi) / \tau_{n}\right) d \xi \\
= & \left\langle q_{v}\right\rangle_{\mathrm{p}} T_{\infty} \frac{a_{n}}{\tau_{n}} \sum_{m=0}^{M-1} \int_{m t_{\mathrm{PRF}}}^{m t t_{\mathrm{PRF}}+t_{\mathrm{PW}}} \exp \left(-(t-\xi) / \tau_{n}\right) d \xi \\
= & \left\langle q_{v}\right\rangle_{\mathrm{p}} T_{\infty} a_{n}\left(\exp \left(t_{\mathrm{PW}} / \tau_{n}\right)-1\right) \exp \left(-t / \tau_{n}\right) \\
& \cdot \sum_{m=0}^{M-1} \exp \left(m t_{\mathrm{PRF}} / \tau_{n}\right) \\
= & \left\langle q_{v}\right\rangle_{\mathrm{p}} T_{\infty} a_{n} \frac{1-\exp \left(t_{\mathrm{PW}} / \tau_{n}\right)}{1-\exp \left(t_{\mathrm{PRF}} / \tau_{n}\right)} \exp \left(-t / \tau_{n}\right) \\
& \cdot\left(\exp \left(M t_{\mathrm{PRF}} / \tau_{n}\right)-1\right)
\end{aligned}
$$

The quantity $M t_{\mathrm{PRF}}$ is approximately the exposure time $t$ for pulsed ultrasound exposure times greater than several pulses. Therefore, the temperature increase resulting from exposure to a pulsed ultrasound field for $t<M t_{\mathrm{PRF}}$ is

$$
T_{p}(t)=\left\langle q_{v}\right\rangle_{\mathrm{p}} T_{\infty} a_{n} \frac{1-\exp \left(t_{\mathrm{PW}} / \tau_{n}\right)}{1-\exp \left(t_{\mathrm{PRF}} / \tau_{n}\right)}\left(1-\exp \left(-t / \tau_{n}\right)\right)
$$

The pulse width $t_{\mathrm{PW}}$ and pulse repetition period $t_{\mathrm{PRF}}$ are several orders of magnitude less than the thermal response time constants $\tau_{n}$ in the summation, even for very small specimens and/or beam sizes so that

$$
\frac{1-\exp \left(t_{\mathrm{PW}} / \tau_{n}\right)}{1-\exp \left(t_{\mathrm{PRF}} / \tau_{n}\right)} \approx \frac{t_{\mathrm{PW}}}{t_{\mathrm{PRF}}}=t_{\mathrm{PW}} \times P R F
$$

Upon reintroducing the summation over $n$, the temperature elevation for $t<M t_{\mathrm{PRF}}$ is then

$$
T_{p}(t) \approx\left\langle q_{v}\right\rangle_{\mathrm{p}} T_{\infty} \times t_{\mathrm{PW}} \times P R F \sum_{n=1}^{N} a_{n}\left(1-\exp \left(-t / \tau_{n}\right)\right)
$$

The rate of heat generation per unit volume, time-averaged over a single pulse $\left\langle q_{v}\right\rangle_{\mathrm{P}}$, is related to the acoustic field quantities from Eq. (3) as

$$
\left\langle q_{v}\right\rangle_{\mathrm{p}}=2 \alpha \frac{\left\langle p^{2}\right\rangle}{\rho c_{o}}=2 \alpha \frac{1}{t_{\mathrm{PW}}} \int_{0}^{t_{\mathrm{PW}}} \frac{p^{2}(t)}{\rho c_{o}} d t
$$

The integral term is the spatial-peak, pulse-average (SPPA) intensity $I_{\mathrm{SPPA}}$ for a pulsed ultrasound field. ${ }^{16)}$ The temperature increase resulting from the pulsed ultrasound exposure is then

$$
\begin{aligned}
T_{p}(t) \approx & \frac{2 \alpha}{\rho C_{p}} \phi \times I_{\mathrm{SPP}} \times t_{\mathrm{PW}} \times P R F \\
& \cdot \sum_{n=1}^{N} a_{n}\left(1-\exp \left(-t / \tau_{n}\right)\right)
\end{aligned}
$$

where only those time constants $\tau_{n}$ for all $t_{\mathrm{PW}} / \tau_{n}$, $t_{\mathrm{PRF}} / \tau_{n} \ll 1$ are included in the above summation. The product $I_{\mathrm{SPPA}} \times t_{\mathrm{PW}} \times P R F$ is defined for medical ultrasound fields as $I_{\mathrm{SPTA} \cdot{ }^{16)}}$ Upon substituting this into Eq. (17), the temperature elevation resulting from a pulsed ultrasound exposure would then be the same as that measured for a $c w$ exposure for identical $I_{\mathrm{SPTA}}$, viz., Eq. (7).

\section{DISCUSSION}

The heat generation rate per unit volume has been assumed in the preceding development to be proportional to the infinitesimal acoustic amplitude absorption coefficient $\alpha$, i.e., the absorption coefficient that would be measured in an ultrasound field with negligible harmonic development. It is welldocumented, however, that some medical diagnostic instruments produce a highly nonlinear acoustic field at the focus. ${ }^{17-19)}$ The ultrasonic absorption or rate of heat generation in such fields can be significantly greater than that in a linear acoustic field. ${ }^{4,20-23)}$ However, it is seen from Eq. (6) that the above development holds for any field in which $\left\langle q_{v}\right\rangle$ is proportional to $I_{\mathrm{SPTA}}$. Likewise, though Eq. (2) applies to absorption in soft tissue or to fluid media generally, since a medium with zero shear viscosity must be assumed to deduce Eq. (2) for $\left\langle q_{v}\right\rangle$, the above results should apply to hard tissue such as fetal bone as well, if the temperature increase in the tissue is directly proportional to the incident $I_{\mathrm{SPTA}}$ in the surrounding fluid. The temperature elevation in fetal bone has been found to vary linearly with $I_{\mathrm{SPTA}}$ of the incident field in the coupling fluid. ${ }^{15)}$ Then, the temperature increase in the fetal bone should be proportional to $I_{\mathrm{SPTA}}$ independent of the type of exposure, i.e., $c w$ or plused.

\section{ACKNOWLEDGEMENT}

The authors gratefully acknowledge the partial support of this work by a grant from the NIH.

\section{REFERENCES}

1) L. A. Frizzell, C. A. Linke, E. L. Carstensen, and C. W. Fridd, "Thresholds for focal lesions in rabbit kidney, liver, and testicle," IEEE Trans. Biomed. Eng. BME-24, 393-396 (1977).

2) L. A. Frizzell, "Threshold dosages for damage to mammalian liver by high intensity focused ultrasound," IEEE Trans. Ultrason. Ferroelectr. Freq. Control UFFC-35, 578-581 (1988). 


\section{J. L. DREWNIAK and F. DUNN: PULSED AND CW ULTRASOUND TEMPERATURE ELEVATION}

3) F. J. Fry, G. Kossoff, R. C. Eggleton, and F. Dunn, "Threshold ultrasonic dosages for structural changes in the mammalian brain," J. Acoust. Soc. Am. 48, 1413-1417 (1970).

4) D. Dalecki, E. L. Carstensen, K. J. Parker, and D. R. Bacon, "Absorption of finite amplitude focused ultrasound," J. Acoust. Soc. Am. 89, 2435-2447 (1991).

5) H. S. Carslaw and J. C. Jaeger, Conduction of Heat in Solids (Oxford University Press, Inc., New York, 1959).

6) N. M. Ozisik, Heat Conduction (John Wiley \& Sons, Inc., New York, 1980).

7) V. Abraham, M. C. Ziskin, and S. Heynen, "Temperature elevation in the rat fetus due to ultrasound exposure," Ultrasound Med. Biol. 15, 443449 (1989).

8) K. I. Carnes, J. L. Drewniak, and F. Dunn, "In utero measurements of ultrasonically induced fetal mouse temperature increases," Ultrasound Med. Biol. 17, 373-382 (1991).

9) M. E. Lyons and K. J. Parker, "Absorption and attenuation in soft tissues: II-experimental results," IEEE Trans. Ultrason. Ferroelectr. Freq. Control UFFC-35, 511-521 (1988).

10) W. L. Nyborg, "Heat generation by ultrasound in a relaxing medium," J. Acoust. Soc. Am. 70, 310-312, 1981.

11) T. J. Cavicchi and W. D. O'Brien, Jr., "Heat generated by ultrasound in an absorbing medium," J. Acoust. Soc. Am. 76, 1244-1245 (1984).

12) K. Beissner, "On the plane wave approximation of acoustic intensity," J. Acoust. Soc. Am. 71, 14061411 (1982).

13) J. L. Drewniak, L. A. Frizzell, and F. Dunn, "Errors resulting from finite beamwidth and sample dimensions in the determination of the ultrasonic absorption coefficient," J. Acoust. Soc. Am. 88, 967-977 (1990).

14) F. Dunn, P. D. Edmonds, and W. J. Fry, "Absorption and dispersion of ultrasound in biological media," in Biomedical Engineering, H. P. Schwan, Ed. (McGraw-Hill Book Company, New York, 1969), Chap. 3, pp. 205-332.

15) J. L. Drewniak, K. I. Carnes, and F. Dunn, "In vitro ultrasonic heating of fetal bone," J. Acoust. Soc. Am. 86, 1254-1258 (1989).

16) G. R. Harris, "A discussion of procedures for ultrasonic intensity and power calculations from miniature hydrophone measurements," Ultrasound Med. Biol. 11, 803-817 (1985).

17) F. A. Duck, H. C. Starritt, J. D. Aindow, M. A. Perkins, and A. J. Hawkins, "The output of pulseecho ultrasound equipment: A survey of powers, pressures, and intensities," Br. J. Radiol. 58, 9891001 (1985).

18) K. J. Parker, "Observation of nonlinear acoustic effects in a B-Scan imaging instrument," IEEE
Trans. Sonics Ultrason. SU-32, 4-8 (1985).

19) H. C. Starritt, M. A. Perkins, F. A. Duck, and V. F. Humphrey, "Evidence for ultrasonic finite amplitude distortion in muscle using medical equipment," J. Acoust. Soc. Am. 77, 302-306 (1985).

20) E. L. Carstensen, S. A. Becroft, W. K. Law, and D. B. Barbee, "Finite amplitude effects on the thresholds for lesion production in tissues by unfocused ultrasound," J. Acoust. Soc. Am. 70, 302309 (1981).

21) E. L. Carstensen, N. D. McKay, D. Dalecki, and T. G. Miur, "Absorption of finite amplitude ultrasound in tissues," Acustica 51, 116-123 (1982).

22) F. J. Fry, K. A. Dines, C. R. Rielly, and S. A. Goss, "Losses in tissue associated with finite amplitude ultrasound transmission," Ultrasound Med. Biol. 15, 481-497 (1989).

23) S. A. Goss and F. J. Fry, "Nonlinear acoustic behavior in focused ultrasonic fields: observations of intensity dependent absorption in biological tissue," IEEE Trans. Sonics Ultrason. SU-28, 21-26 (1981).

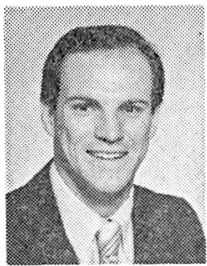

James L. Drewniak was born in Beach, ND, on July 13, 1962. He received the B.S. (highest honors), $\mathrm{M}$. S. and Ph.D. degrees in electrical engineering from the University of Illinois, Urbana-Champaign in 1985 , 1987, and 1991, respectively. From 1985 to 1987 he was a Graduate Research Assistant in the Electromagnetics Laboratory at the University of Illinois investigating broadband, traveling wave antennas. From 1987-1991 he was a Graduate Research Assistant in the Bioacoustics Laboratory at the University of Illinois investigating ultrasonic properties of hard and soft tissues. In July 1991 he joined the Electrical Engineering Department at the University of Missouri-Rolla as an Assistant Professor. His research interests include numerical electromagnetic and acoustic analysis, antennas, and ultrasonic tissue characterization. Dr. Drewniak is a member of the Institute for Electrical and Electronics Engineers, the Acoustical Society of America, Eta Kappa Nu, Tau Beta Pi, and Phi Kappa Phi.

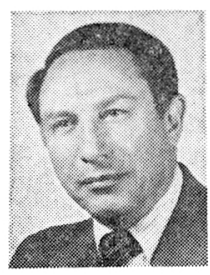

Floyd Dunn was born in Kansas City, MO, on April 14, 1924. He took the B.S., M.S., and Ph.D. degrees in 1949, 1951, and 1956, respectively, all in electrical engineering at the University of Illinois, Urbana, where he specialized in bioacoustics. $\mathrm{He}$ holds joint appointments as Professor of Electrical Engineering, of Bioengineering, and of Biophysics, and is Director of the Bioacoustics Research Laboratory at the University of Illinois, and he was a Visiting Professor at Tohoku University, 1982 and 1989/ 90. His research interests deal with all aspects of the 
interaction of ultrasound and biological media. Professor Dunn is a member of the National Academy of Sciences and the National Academy of Engineering; a Fellow of the Acoustical Society of America (President 1985/6), of the American Institute of Ultrasound in Medicine, of the Institute of Electrical and Electronic Engineers, of the American Association for the Advance- ment of Sciences, and of the Institute of Acoustics; and an Honorary Member of the Japan Society of Ultrasound in Medicine. He has been awarded the Silver Medal of the Acoustical Society of America, the Wm. J. Fry and the Joseph H. Holmes Awards of the American Institute of Ultrasound in Medicine, and the Medal of Special Merit of the Acoustical Society of Japan. 